$$
\Xi(M)=\Phi\left(\varphi_{M}\right)-\frac{1}{\gamma} \log p_{i},
$$

in wlich

$$
\begin{aligned}
& \Xi(M)=-\frac{\gamma-1}{2} \int M^{3}\left(1+\frac{\gamma-1}{2} M^{2}\right)^{-1} d M, \\
& \Phi\left(\varphi_{M}\right)=\frac{\gamma-1}{\gamma+1} \int f\left(\varphi_{M}\right) d \varphi_{M} .
\end{aligned}
$$

(In uniplanar flow, explicit formulae for potential and stream functions can be derived for both irrotational $\mathbf{M}$ and some irrotational $\mathbf{W}$ flows. We have not ascertained whether these formulae represent more than the well-known elementary radial and vortex flows.)

If $\mathrm{W}=\nabla \varphi_{W}$, then $\nabla p_{t}=0$, a case discussed by Vazsonyi $\mathrm{i}^{4}$ in the less appropriate $\mathrm{V}$ language. Flows with constant stagnation pressure but variable entropy and stagnation temperature from one streamline to another may be of immediate practical interest. For example the effect of variable chamber gas temperature upon thrust coefficient of a rocket with "perfect" nozzle can be computed. This calculation is possible because irrotational $\mathbf{V}$ flow is the special case of irrotational $\mathbf{W}$ flow which occurs when not only $p_{t}$ but also $S$ and therefore $T_{t}$ are constant throughout the flow $\left(\nabla S=\nabla T_{t}=0\right.$ is implied by $\left.\mathbf{V}=\nabla \varphi_{V}\right)$. Accordingly the same partial differential equation (derived from the continuity equation (1.10)) is satisfied by both $\varphi_{W}$ and its isentropic form $\varphi_{V}$,

$$
\sum_{i=1}^{3}\left[1-W^{2}-\frac{2}{\gamma-1}\left(\frac{\partial \varphi_{w}}{\partial x_{i}}\right)^{2}\right] \frac{\partial^{2} \varphi_{w}}{\partial x_{i}{ }^{2}}-\frac{4}{\gamma-1} \sum_{i>j=1}^{3} \frac{\partial \varphi_{w}}{\partial x_{i}} \frac{\partial \varphi_{w}}{\partial x_{j}} \frac{\partial^{2} \varphi_{w}}{\partial x_{i} \partial x_{j}}=0 .
$$

\title{
LINEARIZATION OF SOLUTIONS IN SUPERSONIC FLOW*
}

\section{By JOHN W. TUKEY (Bell Telephone Laboratories and Princeton University)}

1. Introduction. The equations governing flow at supersonic speeds are believed to be well known, but the difficulties of calculating exact solutions are so great that approximate solutions are the aim of the present and foreseeable future. Two main approaches to such approximate solutions are commonly considered:

(a) the calculation of numerical approximations by high-speed calculators,

(b) simplification of the equations and explicit solution of the simplified equations.

Of course the first route requires the high-speed calculating machines which are now in sight, but not yet available.

It is the purpose of this note to propose and exemplify a third approach which may partly replace (b) and frequently supplement (a), namely

(c) simplification of the dependence of the answers on one or more parameters.

2. Is linearization of solutions mathematically justified? The first objection which many would raise against "linearizing the solutions" as a substitute for "linearizing

\footnotetext{
* Received Feb. 15, 1947.
} 
the equations" is its matrematical justification. The justification of the former would seem to be at least as good as the latter. In order to make this clear, let us look at the situation in more detail.

A problem is believed to be adequately stated by a complex set of equations, which we may symbolize by

$$
F(x, \lambda)=0,
$$

where $\lambda$ is a parameter. The classical procedure is to replace the complex set of equations by a simpler set, frequently composed of linear equations, which we may write

$$
L(x, \lambda)=0,
$$

and then to solve these equations exactly to obtain

$$
x=g(\lambda) .
$$

We must interpret this solution as an approximate solution of the original equations. What justification might we have? The only possibilities seem to be

(a) a theorem that the solutions of (2) are close to the solutions of (1),

(b) empirical evidence that, in particular cases, the solutions of (2) are close to the solutions of (1),

(c) mathematical intuition,

(d) physical intuition.

The author knows of no case in supersonic flow where the very desirable justification of type (a) exists.

On the other hand, suppose that (1) can be solved for, say, two values of $\lambda$, so that we know $x_{1}$ and $x_{2}$, where

$$
F\left(x_{1}, \lambda_{1}\right)=0=F\left(x_{2}, \lambda_{2}\right),
$$

and suppose further that we believe that we can approximate the dependence of $x$ on $\lambda$ over a certain range by one of, say, a two-parameter family of functions,

$$
x(\lambda)=h(\lambda ; A, B) .
$$

We can then use the two known cases to determine the parameters and find an approximate solution

$$
x=h\left(\lambda ; A_{0}, B_{0}\right)
$$

of (1). What justification might we have? The only possibilities seem to be

(a) a theorem that the solution of (1) depends on $\lambda$ in a way close to one of the functions (4),

(b) empirical evidence that, in particular cases, the solutions of (1) do behave like one of the functions (4),

(c) mathematical intuition,

(d) physical intuition.

The author knows of no case in supersonic flow where the very desirable justification of type (a) exists.

Thus the two ways of "linearizing" seem equally justified. The first appeals to some because the solution used is exactly a solution of (2). There is no reason, how- 
ever, why this should be more esthetic than the fact that the other approximation is in exactly the form 4 .

3. A linearization for the region of attacked shock waves. In the range of supersonic speeds safely away from sonic or hypersonic speeds, there seems to be some evidence that a linearization of the form

$$
A p+B q
$$

where $q$, the dynamic pressure, is given by

$$
q=\frac{\gamma}{2} M^{2} p
$$

applies approximately to many averaged quantities, such as lifts, drags, etc., for well streamlined bodies. Some empirical evidence is presented in the next section. It is believed that, when the two points needed to determine $A$ and $B$ are known from theory or experiment, the use of this linearization will frequently give results preferable to those obtained by solving a linearized problem.

4. The flow around an axially-symmetrical cone. The theoretical problem of flow around a cone was solved by Taylor and Maccoll ${ }^{1}$ who computed the drag or pressure coefficient for several cases. These calculations were refined and extended by Maccoll. ${ }^{2}$

A linearized theory was previously presented by v. Kármán and Moore. ${ }^{3}$

The present paragraph is concerned with the empirical linearization

$$
C_{p} \doteq\left(0.083+\frac{0.096}{M^{2}}\right)\left(\frac{\theta_{s}}{10^{\circ}}\right)^{1.69}, \quad\left(\theta_{s}=\text { half angle of cone }\right)
$$

The form and constants of this approximation are purely empirical, and are principally based on Maccoll's theoretical values.

Figure 4 on p. 309 of the paper by Taylor and Maccoll ${ }^{1}$ shows that the linearized equations give rise to errors of 10 to 25 per cent over substantial portions of the range, while the following table shows that the empirical formula gives agreement to better than 5 per cent over a range of half angles from $10^{\circ}$ to $55^{\circ}$ (Maccoll estimates $57.4^{\circ}$ as the maximum half angle for conical flow) and over a range of Mach numbers for each angle extending down to within about 0.1 of the minimum Mach number for conical flow (attached shock-wave).

The fit is not perfect, and it is clear that the empirical formula cannot replace the exact solution for all purposes. However, as a means of interpolating between known values, it clearly serves a useful purpose.

Summary. There is theoretical and empirical justification for "linearizing the answer" rather than "linearizing the equations" in some fields of supersonic flow.

${ }^{1}$ G. I. Taylor and J. W. Maccoll, The air pressure on a cone moving at high speeds, Proc. Roy. Soc. London (A) 139, 278-311 (1933).

${ }^{2} \mathrm{~J}$. W. Maccoll, The conical shock wave formed by a cone moving at a high speed, Proc. Roy. Soc. London (A) $159,459-472$ (1937).

${ }^{3}$ Th. von Kármán and N. B. Moore, Resistance of slender bodies movir $g$ with supersonic velocities, with special reference to projectiles, Tr. Amer. Soc. Mech. Eng. 54, 303-310 (1932); for further discussion see Taylor and Maccoll, loc. cit., p. 30. 
TABle 1. Supersonic Pressure Coefficient of Unyawed cone

\begin{tabular}{|c|c|c|c|c|}
\hline \multirow{2}{*}{$\begin{array}{l}\text { Semiangle of Cone } \\
\text { (Min. Mach No.2) }\end{array}$} & \multirow{2}{*}{$\begin{array}{l}\text { Mach } \\
\text { Number }\end{array}$} & \multicolumn{2}{|c|}{ Pressure Coefficient ${ }^{1}$} & \multirow{2}{*}{$\begin{array}{l}\text { Per Cent } \\
\text { Deviation }\end{array}$} \\
\hline & & Quadrature $^{3}$ & Empirical ${ }^{+}$ & \\
\hline $10^{\circ}$ & 1.091 & $.156^{5}$ & .161 & +5 \\
\hline \multirow{5}{*}{$(1.04)$} & 1.403 & .130 & .131 & +1 \\
\hline & 1.81 & .108 & .112 & +4 \\
\hline & 2.39 & .096 & .100 & +4 \\
\hline & 3.33 & .090 & .092 & +2 \\
\hline & 5.46 & .076 & .086 & +13 \\
\hline $20^{\circ}$ & 1.314 & .446 & .447 & +0 \\
\hline \multirow[t]{6}{*}{$(1.18)$} & 1.555 & .374 & .394 & +5 \\
\hline & 1.65 & .356 & .382 & +7 \\
\hline & 2.13 & .318 & .333 & +5 \\
\hline & 2.86 & .296 & .306 & +3 \\
\hline & 4.17 & .268 & .286 & +7 \\
\hline & 9.74 & .256 & .271 & +6 \\
\hline $30^{\circ}$ & 1.514 & .814 & .800 & -2 \\
\hline \multirow[t]{6}{*}{$(1.46)$} & 1.639 & .730 & .760 & +4 \\
\hline & 2.05 & .636 & .678 & +7 \\
\hline & $2.71^{\circ}$ & .598 & .614 & +3 \\
\hline & 3.16 & .570 & .593 & +4 \\
\hline & 3.85 & .556 & .572 & +3 \\
\hline & 7.84 & .524 & .542 & +3 \\
\hline $40^{\circ}$ & 2.03 & 1.098 & 1.106 & +1 \\
\hline \multirow[t]{4}{*}{ (1.95) } & 2.22 & 1.036 & 1.067 & +3 \\
\hline & 2.84 & 0.948 & 0.988 & +4 \\
\hline & 4.20 & 0.904 & 0.921 & +0 \\
\hline & 5.86 & 0.890 & 0.893 & \\
\hline $45^{\circ}$ & 2.38 & 1.316 & 1.270 & -4 \\
\hline \multirow[t]{4}{*}{$(2.3)$} & 2.72 & 1.182 & 1.219 & +4 \\
\hline & 3.76 & 1.104 & 1.140 & +4 \\
\hline & 4.89 & 1.084 & 1.105 & +2 \\
\hline & 8.01 & 1.068 & 1.073 & +1 \\
\hline & 3.15 & 1.440 & 1.407 & -2 \\
\hline \multirow[t]{4}{*}{ (3.1) } & 3.33 & 1.374 & 1.391 & +1 \\
\hline & 3.81 & 1.330 & 1.360 & +2 \\
\hline & 4.75 & 1.296 & 1.325 & +2 \\
\hline & 7.11 & 1.266 & 1.289 & +2 \\
\hline $55^{\circ}$ & 5.59 & 1.552 & 1.535 & -1 \\
\hline \multirow[t]{2}{*}{$(5.5)$} & 6.38 & 1.516 & 1.522 & 0 \\
\hline & 7.56 & 1.496 & 1.510 & +1 \\
\hline
\end{tabular}

1 Given in American units-Maccoll's values have been doubled.

2 Minimum for conical flow and attached shock wave.

${ }^{3}$ Taken from Maccoll, Proc. Roy. Soc. 159 (1937) pp. 461 and 469, also 139 (1933) p. 285.

4 Calculated by formula $\left({ }^{*}\right)$ above.

- This value is undoubtedly too low by 10 to 20 per cent. 
The linearization

$$
\text { (drag, lift, etc.) }=A p+B q
$$

is proposed for use in the region of attached shock waves.

\section{BOOK REVIEWS}

Tables of spherical Bessel functions. Prepared by the Mathematical Tables Project, National Bureau of Standards. Vol. 1. Columbia University Press, New York, 1947. xxviii +375 pp. $\$ 7.50$.

In this volume the functions $\sqrt{\pi / 2 x} J_{\nu}(x)$ are tabulated for the half-odd-integer values of $\nu$, $-27 / 2 \leqq \nu \leqq 27 / 2$. For $0 \leqq x \leqq 10.0$, the functions are tabulated to eight or more significant figures at intervals of .010 . For $10 \leqq x \leqq 25$, the tables contain seven significant figures and the argument interval is.10. A table of the function $x^{-\nu+1 / 2} \sqrt{\pi / 2 x} J_{\nu}(x)$ is also included for argument intervals of .01. The range of argument is: for $\nu=-\frac{1}{2}, \pm 5 / 2, \pm 7 / 2: 0 \leqq x \leqq 1$; for $\nu= \pm 3 / 2: 0 \leqq x \leqq .50$; for $\nu= \pm 9 / 2, \pm 11 / 2, \cdots$, $\pm 15 / 2: 0 \leqq x \leqq 1.5 ;$ for $\nu= \pm 17 / 2, \cdots, 23 / 2: 0 \leqq x \leqq 2$; and for $\nu= \pm 25 / 2, \pm 27 / 2: 0 \leqq x \leqq 2.5$.

G. F. CARRIER

Introduction to aerodynamics of a compressible fluid. By Hans Wolfgang Liepmann and Allen E. Puckett. Galcit Aeronautical Series. John Wiley \& Sons, Inc., New York, 1947. ix +262 pp. $\$ 4.00$.

The better known works on the aerodynamics of compressible fluids are 1) Ackeret's and 2) Busemann's articles in the Handbuch der Physik and the Handbuch der Experimentalphysik; 3) Bateman's article in the Report of the Committee on Hydrodynamics of the National Research Council; 4) Taylor and Maccoll's article in Durand's Aerodynamic Theory; 5) papers collected in the Proceedings of the Fifth Volta Congress and 6) Sauer's rather recent book. The first three, although excellent summaries of information up to the dates of their publication are now outdated. The fourth is masterly in its choice of subjects and exposition but may be too difficult for a beginner in the subject due to its briefness. The fifth contains truly a wealth of good papers covering the whole field, but unfortunately it is out of print. The sixth and last one is written by a mathematician from the point of view of a mathematician.

Therefore for aeronautical engineers now entering upon a study of aerodynamics of compressible fluids, there was a great need for an introductory book with a clear exposition of the physical aspects and the recent advancement of the subject. This need is believed to be admirably satisfied by Liepmann and Puckett's book. Of course, as the title indicates, the book is not a compendium of all available data on compressible flows, and for engineers who are desperately looking for design information, this book will be a disappointment. However, for those who are interested in a clear understanding of the phenomena of compressible flow, the outstanding feature of this book is the constant emphasis on the interplay between theory and physical facts. The authors always try to present a description before going into a calculation. Thus the gist of a problem is not lost in a maze of equations. It also is evident that the authors are always aware of the limitations imposed by simplifying assumptions which are, of course, necessary in order to make the problem tractable, and such limitations are always pointed out in due time. The reader is thus saved from the various pitfalls in applying the results of a theory. The authors' research efforts in compressible flow are also evident in the book because not only are known facts expounded, but difficult questions in such subjects of current interest as condensation shock, Mach reflection, influence of viscosity in transonic flows are also discussed.

However there are occasions in the book where the authors leave an inquisitive mind unsatisfied. For instance, the treatment in Part II on two- and three-dimensional motions is uneven in its pace.-While great details of ccmputations are given in deriving the basic perturbation equations, no method of solving these equations or example is given, so that reader could have a more concrete understanding of the principles involved. Then for condensation shocks, there is the question of the relative importance of dust 